\title{
The Third Workshop on Compact Steep Spectrum and GHz-Peaked Spectrum Radio Sources: Kerastari, Tripolis, Greece, 29-31 May 2002
}

\author{
Tasso Tzioumis
}

Australia Telescope National Facility, Epping 1710, Australia

tasso.tzioumis@atnf.csiro.au

Recent work has identified the GHz Peaked Spectrum (GPS) and Compact Steep Spectrum (CSS) radio sources as the most likely candidates for the progenitors of the large scale radio sources. The GPS and CSS sources are powerful but compact radio sources whose spectra are generally simple and convex with peaks near $1 \mathrm{GHz}$ and $100 \mathrm{MHz}$ respectively. The GPS sources are entirely contained within the extent of the narrow line region $(<1 \mathrm{kpc})$ while the CSS sources are contained entirely within the host galaxy $(<15 \mathrm{kpc})$.

Current models for the evolution of powerful radio galaxies suggest that these sources propagate from the $10 \mathrm{pc}$ to Mpc scales at roughly constant velocity through an ambient medium which declines in density while the sources decline in radio luminosity. The observed number densities of powerful radio sources as a function of linear size (from tens of parsecs to hundreds of $\mathrm{kpc}$ ) are consistent with such a scenario. Thus, the GPS and CSS sources provide both probes of the ISM of the host galaxy and constraints on the physics of radio galaxy evolution.

Rapid progress has been made in our understanding of GPS and CSS sources through the combination of high resolution radio and optical imaging as well as IR and X-ray observations. This workshop brought together observers from across the spectrum with theorists for an informal and stimulating exchange of ideas and results.

About 40 scientists from around the world participated in the Workshop with many oral and poster presentations, as outlined in the accompanying program. All the presentations are published in these proceedings and are also briefly summarised below by Lo Woltjer, as part of his closing remarks at the workshop.

This workshop was the third in a series following the 1990 meeting in Dwingeloo and the 1996 meeting in Leiden, both in The Netherlands. The Workshop was held in the Greek village of Kerastari, the birthplace of the Local Organising Committee (LOC) chair, Tasso Tzioumis. It is a tiny picturesque village, nestled in the mountains of ancient Arcadia near the town of Tripolis, in the Peloponese, the southernmost part of Greece.

The success of the workshop is in large part due to the enthusiastic support of the local community at all levels. Such a workshop has never been held before in this part of the world and the locals were fascinated, if somewhat bewildered. The villagers adopted the Workshop as their own and turned out in force to celebrate and to help. The local Municipality of Valtetsi sponsored the workshop and provided both material and moral support. The speech of the Mayor, reproduced below, reflects the feelings of the locals together with their fascination, support and good wishes. The workshop was also supported by the regional government of the Prefecture of Tripolis and the Municipality of Tripolis.

The local issues and day-to-day running of the workshop were ably covered by the LOC, which was chaired by Tasso Tzioumis (Australia) and included Antonis Polatidis (Germany) and John Seiradakis (Greece).

The Workshop owes its scientific breadth and success to the untiring efforts of the international Scientific Organising Committee (SOC). This committee was chaired by Chris O'Dea (USA) and included Geoff Bicknell (Australia), Wim de Vries (USA), Carla Fanti (Italy), Roberto Fanti (Italy), Anton Koekemoer (USA), Richard Schilizzi (Netherlands), Ignas Snellen (UK) and Tasso Tzioumis (Australia).

The publication of the proceedings of the workshop presented here was made possible through the generous sponsorship of the Publications of the Astronomical Society of Australia (PASA) and the efforts of its editors Michelle Storey and Richard Hecker. They were greatly assisted in this endeavour by a guest editorial committee chaired by Tasso Tzioumis and included Wim de Vries, Anton Koekemoer and Ignas Snellen. They guided the long and sometimes tortuous reviewing and revising process for about eight months. It has now culminated in an excellent issue covering $100 \%$ of the workshop presentations!

The efforts of all these people made this not just another workshop but an event. In spontaneous feedback from some of the participants one mentions that 'Your meeting has reset the high end of the scale for such events.' And the local community is still basking in the excitement.

Finally, I'd like to express my personal thanks and gratitude to all that participated and helped to make the workshop such a success. I feel deeply honoured that so many of my colleagues participated in this event in my small village and that my fellow villagers and local community supported this so wholeheartedly.

And I'd like to dedicate this workshop to my dearest daughter Eirini Tzioumis, who, despite severe health problems, encouraged and supported me throughout this endeavour and made the pilgrimage to the village for the workshop.

Tasso Tzioumis April 2003 


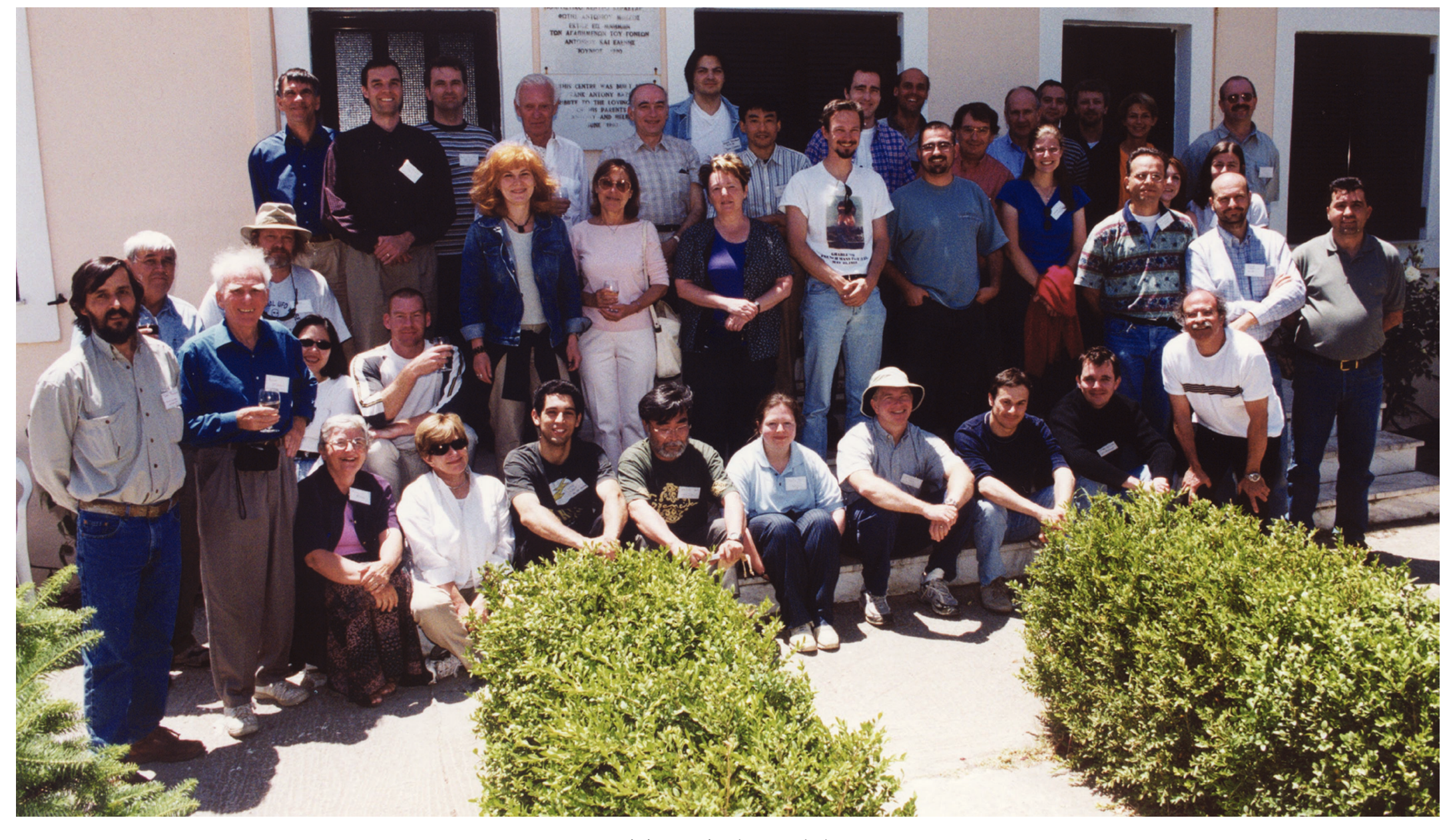

Participants in the Workshop. 
Table 1. Workshop program for 29-31 May 2002

\section{Wednesday morning}

New Samples

Wednesday afternoon: Radio Properties

Thursday morning Host Galaxies, Optical and X-rays

Friday morning: Environment

Friday afternoon: Evolution

Posters
Radio Properties of GPS and CSS

Sources: Galaxies versus Quasars

High Frequency Peakers

Radio-Excess IRAS Galaxies:

Low Power CSS/GPS Sources?

Radio Spectral Variability of the Nearby

GPS Galaxy PKS 1718-649

Variability of GPS Sources

Proper Motions in Compact Symmetric Objects Spectral Ages of CSOs and CSS Sources

Polarimetry of GPS and CSS Sources

Properties of Radio Source Host Galaxies Emission Line Properties of GPS/CSS Galaxies Optical and UV Spectral Diagnostics for GPS and CSS Sources

The Impact of the Early Stages of Radio Source Evolution on the ISM of the Host Galaxies Jet-Cloud Interactions in Compact Steep Spectrum Radio Sources

Beppo SAX X-ray Observations of PKS 1934-63 and S5 1946+708

RM and Opacity Asymmetry in 2134+004 Dense Plasma Torus in the GPS Galaxy NGC 1052

$H_{I}$ Absorption in GPS/CSS Sources

$X$-ray Emission from GPS/CSS Sources

The Shroud Around the 'Compact, Symmetric' Radio Jets in NGC 1052

GPS and CSS Sources-Theory and Modelling

Constraining the Evolution of Young

Radio-Loud AGN

A Dynamical Model for the Evolution of Hot Spots in Powerful Radio Sources

Symmetry Parameters of CSS Sources: Evidence of Fuelling?

A Population Study of Faint Compact Steep Spectrum Radio Sources: Self-Similar Growth III Zw 2: Evolution of a Radio Galaxy in a Nutshell

Location of Weak CSS Sources on the Evolutionary Path of Radio-Loud AGN

Concluding Remarks

Luminosity Evolution of Double Radio Sources Extreme Emission Line Outflows in the GPS Source 4C 12.50 (PKS 1345+12)

HST/STIS Spectroscopy of CSS Sources: Kinematics and Ionisation of the Aligned Nebulae

Two-Sided Radio Jets in B1524-136

The Polarised Bent Jet of $3 C 43$ Weak CSS Sources from the FIRST Survey

$1245+676-A$ CSO/GPS Source being an Extreme Case of a Double-Double Structure Search for CSS and GPS Candidates from the Peacock and Wall Catalogue

The Extraordinary Radio Galaxy MRC B1221-423

VLBA Observations of Bright High Frequency Peakers

Free-Free Absorption and the Unified State
C. Stanghellini

D. Dallacasa

C. L. Drake, P. J. McGregor, G. V. Bicknell and M. A. Dopita

S. J. Tingay and M. de Kool

D. L. Jauncey, E. A. King, H. E. Bignall, J. E. J. Lovell, L. Kedziora-Chudczer, A. K. Tzioumis, S. J. Tingay,

J.-P. Macquart and P. M. McCulloch

A. G. Polatidis and J. E. Conway

M. Murgia

W. D. Cotton, D. Dallacasa, C. Fanti, R. Fanti,

A. R. Foley, R. T. Schilizzi, R. Spencer,

D. J. Saikia and S. Garrington

W. de Vries

A. M. Koekemoer, C. P. O'Dea and S. A. Baum

M. A. Dopita

R. Morganti, C. N. Tadhunter, T. A. Oosterloo,

J. Holt, A. Tzioumis and K. Wills

C. P. O’Dea, W. H. de Vries, A. M. Koekemoer,

S. A. Baum, D. J. Axon, P. D. Barthel, A. Capetti,

R. Fanti, R. Gelderman, R. Morganti and C. N. Tadhunter

L. Woltjer, G. Risaliti and M. Salvati

M. Mutoh, M. Inoue, S. Kameno and K. Asada

S. Kameno, M. Inoue, K. Wajima,

S. Sawada-Satoh and Z.-Q. Shen

Y. Pihlstrom, J. Conway and R. Vermeulen

A. Siemiginowska, T. L. Aldcroft, J. Bechtold,

G. Brunetti, M. Elvis and C. Stanghellini

R. C. Vermeulen, E. Ros, K. I. Kellermann,

M. H. Cohen, J. A. Zensus and H. J. van Langevelde

G. V. Bicknell, C. J. Saxton and R. S. Sutherland

I. A. G. Snellen, K.-H. Mack,

R. T. Schilizzi and W. Tschager

M. Perucho and J. M. Martí

D. J. Saikia, S. Jeyakumar, F. Mantovani, C. J. Salter,

R. E. Spencer, P. Thomasson and P. J. Wiita

W. Tschager, R. Schilizzi, H. Rottgering,

I. Snellen, G. Miley and R. Perley

A. Brunthaler, H. Falcke, G. C. Bower, M. F. Aller,

H. D. Aller, H. Teräsranta and T. P. Krichbaum

A. Marecki, R. E. Spencer and M. Kunert

L. Woltjer

J. C. Carvalho and C. P. O'Dea

J. Holt, C. N. Tadhunter and R. Morganti

A. Labiano, C. P. O'Dea, R. Gelderman, W. H. de Vries,

D. J. Axon, P. D. Barthel, S. A. Baum, A. Capetti, R. Fanti,

A. M. Koekemoer, R. Morganti and C. N. Tadhunter

F. Mantovani, M. Bondi, D. J. Saikia, W. Junor,

C. J. Salter and R. Ricci

F. Mantovani, W. Junor, D. J. Saikia and C. J. Salter

A. Marecki, J. Niezgoda, J. Wlodarczak, M. Kunert,

R. E. Spencer and A. J. Kus

A. Marecki, P. D. Barthel, A. Polatidis and I. Owsianik

A. Rossetti, F. Mantovani, C. Fanti and R. Fanti

V. Safouris, R. W. Hunstead and O. R. Prouton

S. Tinti, D. Dallacasa, C. Stanghellini and A. Celotti

S. Kameno, M. Inoue, K. Wajima, S. Sawada-Satoh and Z.-Q. Shen 